Cite this: Phys. Chem. Chem. Phys., 2013,

\title{
Laser synthesis of ligand-free bimetallic nanoparticles for plasmonic applications $\dagger$
}

15, 3075

\author{
R. Intartaglia, ${ }^{\text {*a }}$ G. Das, ${ }^{b}$ K. Bagga, ${ }^{\text {a }}$ A. Gopalakrishnan, ${ }^{b}$ A. Genovese, ${ }^{c}$ M. Povia, ${ }^{c}$ \\ E. Di Fabrizio, ${ }^{b}$ R. Cingolani, ${ }^{a}$ A. Diaspro ${ }^{a}$ and F. Brandi ${ }^{a}$
}

Received 31st July 2012,

Accepted 2nd November 2012

DOI: $10.1039 / \mathrm{c} 2 \mathrm{cp} 42656 \mathrm{k}$

www.rsc.org/pccp

\begin{abstract}
A picosecond laser ablation approach has been developed for the synthesis of ligand-free AuAg bimetallic NPs where the relative amount of $\mathrm{Ag}$ is controlled in situ through a laser shielding effect. Various measurements, such as optical spectroscopy, transmission electron microscopy combined with energy dispersive X-ray spectroscopy and inductively coupled plasma optical emission spectrometry, revealed the generation of homogenous $15 \mathrm{~nm}$ average size bimetallic NPs with different compositions and tunable localized surface plasmon resonance. Furthermore, ligand-free metallic nanoparticles with respect to chemically synthesized nanoparticles display outstanding properties, i.e. featureless Raman background spectrum, which is a basic requirement in many plasmonic applications such as Surface Enhanced Raman Spectroscopy. Various molecules were chemisorbed on the nanoparticle and SERS investigations were carried out, by varying the laser wavelength. The SERS enhancement factor for AuAg bimetallic NPs shows an enhancement factor of about $5.7 \times 10^{5}$ with respect to the flat AuAg surface.
\end{abstract}

\section{Introduction}

Metallic nanoparticles (NPs) are driving the development of diverse applications ranging from biochemical sensors, ${ }^{1,2}$ immunoassays, ${ }^{3,4}$ surface-enhanced spectroscopies ${ }^{5}$ and photovoltaic energy devices. ${ }^{6}$ Further opportunities can be provided by greater structural and compositional complexity arising from the combination of various metals within a single nanostructure. Relative to monometallic NPs, AuAg bimetallic NPs have stimulated further interest because the catalytic properties can be enhanced ${ }^{7,8}$ and localized surface plasmon absorption can be varied continuously between the limits of monometallic Au and Ag NPs. ${ }^{9}$ The tunability of collective resonance over a wide range is required in many applications in which the optical field enhancement associated with surface plasmon is exploited. Indeed, the surface enhanced Raman scattering (SERS) technique relies on surface plasmonic resonances (SPRs), which is a collective oscillation of surface

\footnotetext{
${ }^{a}$ Nanophysics, Istituto Italiano di Tecnologia, via Morego, 30, 16163 Genova, Italy. E-mail: romuald.intartaglia@iit.it

${ }^{b}$ Nanostructure, Istituto Italiano di Tecnologia, via Morego, 30, 16163 Genova, Italy

${ }^{c}$ Nanochemistry, Istituto Italiano di Tecnologia, via Morego, 30, 16163 Genova, Italy

† Electronic supplementary information (ESI) available. See DOI: 10.1039/ c2cp $42656 \mathrm{k}$
}

electrons, generated when laser interacts with the metallic surface. When the molecules reside in the proximity of the metal surface, the enhanced electric field due to the SPRs causes an enhanced Raman signal, enabling single molecule detection (SMD). ${ }^{10}$ The high sensitivity and potentiality for label-free analysis associated with SERS is attractive for applications in physics, chemistry, material science, and biomedical engineering. ${ }^{11,12}$ There has been continuous effort for the fabrication of SERS materials after the reports published by Nie et al. and Kneipp et al. ${ }^{13-17}$ in order to achieve a reliable, reproducible, sensitive, easy in production/sampling and cost effective SERS device.

In this regard, many approaches based on chemical and physical routes have been employed for the synthesis of AuAg bimetallic NPs. The most common one is the co-reduction of the metal precursors in the presence of a stabilizing agent. ${ }^{18,19}$ However, this method of preparation gives rise to toxic NPs due to the presence of an excess of stabilizing ligand (citrate molecules) surrounding the NPs surface. ${ }^{20-22}$ This fact may also limit the adsorption of molecules on the NPs surface for sensing or labeling. Thus, other synthesis methods are required to avoid the presence of particle-associated residual chemicals for potential success of these NPs in the clinical setting. A laserbased approach has been established as an efficient and reliable method for preparing high-purity NP colloids, metallic ${ }^{23}$ semiconductors $^{24-26}$ as well as functional bioconjugated 
NPs, ${ }^{27,28}$ without the use of a chemical reducing agent, which may prevent harmful effects. Up to now, laser-based synthesis of AuAg bimetallic NPs concerned the laser ablation of a single target of gold and silver in liquid, followed by a re-irradiation of the mixed NPs colloidal suspensions. ${ }^{29-31}$ Other groups report the generation of $\mathrm{AuAg}$ bimetallic NPs by laser irradiation of metallic salts. ${ }^{32} \mathrm{AuAg}$ bimetallic NPs compositions are controlled by adjusting both initial concentration of $\mathrm{Ag}$ and $\mathrm{Au}$ colloids and/or the irradiation time. In spite of these successes, the laser-based approach has not fully been explored for the synthesis of ligand-free AuAg bimetallic NPs.

Herein, we discuss the synthesis of AuAg bimetallic NPs by picosecond laser ablation of a Ag target in ligand-free Au NPs colloidal solution and demonstrate that the bimetallic NPs composition is controlled in situ through a laser shielding effect, which is potentially a new laser-based approach for synthesizing bimetallic NPs. Various measurements such as transmission electron microscopy (TEM), optical absorption spectroscopy, Inductively Coupled Plasma Optical Emission Spectrometry (ICP-OES), etc. confirm the generation of bimetallic NPs of different compositions displaying plasmon peak positions varying from 400 to $530 \mathrm{~nm}$. Chemical analysis of the bimetallic NPs solutions reveals a decrease of Ag yield during laser processing as the Au NPs concentration in solution increases allowing the generation of bimetallic NPs of different compositions. We suggest the Ag yield value is associated to the initial Au NPs concentration in solution through the shielding of the incoming laser beam. On the other hand, background Raman measurements on these picosecond laser ablated NPs ( $p s L A-N P s$ ) show no features, confirming the production of NPs free of contaminants. Furthermore, we also investigated the potential use of $p s L A-N P S$ for biosensing applications. In this regard, a low concentrated laser ablated metal NPs solution $\left(1.25 \times 10^{-10} \mathrm{M}\right)$ was used which was later on chemisorbed with the molecules (cresyl violet (CV) $(1 \mathrm{nM})$ and Rd6G $(1 \mu \mathrm{M})$ ) for investigation concerning the SERS application.

\section{Experimental section}

\subsection{Laser synthesis method}

Firstly, ligand-free Au NPs $(8 \mathrm{~nm})$ have been prepared by picosecond laser ablation of Au bulk in deionized water (Fig. S1, ESI + ). Then the final colloidal solution was diluted to obtain several samples with different concentrations. Subsequently, pure $\mathrm{Ag}$ target was ablated in the Au NPs colloidal solution. For each sample, the target was irradiated for equal ablation time, $10 \mathrm{~min}$ at a fluence of $1.8 \mathrm{~J} \mathrm{~cm}^{2}$ chosen for the alloying process (Fig. S2, $\mathrm{ESI} \dagger){ }^{22}$ The color of resulting NPs were found to be dependent on the $\mathrm{Au}$ concentration in solution. The laser system used in this study is a commercial high energy picosecond Nd:YAG laser (continuum leopard) providing a pulse of $60 \mathrm{ps}$ with a maximum pulse energy of $115 \mathrm{~mJ}$ at the fundamental laser wavelength $(1064 \mathrm{~nm})$, a maximum of $55 \mathrm{~mJ}$ at the second harmonic laser wavelength $(532 \mathrm{~nm})$ or a maximum of $35 \mathrm{~mJ}$ at the third harmonic laser wavelength $(355 \mathrm{~nm})$. Experiments have been carried out at a repetition rate of $20 \mathrm{~Hz}$. The laser pulse energy was controlled with a variable attenuator. The laser beam with a diameter of $10 \mathrm{~mm}$ is focused $10 \mathrm{~mm}$ below the target surface using a lens with a focal length of $30 \mathrm{~cm}$. The Ag target $(99.999 \%$ from Alpha Aesar), in the form of a cylinder with a diameter of $6 \mathrm{~mm}$ and a thickness of $10 \mathrm{~mm}$, was placed on the bottom of a quartz cuvette (dimension $10 \times 10 \times 30 \mathrm{~mm}^{3}$ ) and immersed in $1 \mathrm{~mL}$ of Au NPs colloidal solution. The height of the liquid above the target surface was $10 \mathrm{~mm}$. Before each experiment the target was mechanically polished and then washed with the same liquid used for the ablation several times to remove the impurity from the surface. During laser ablation, the target was moved with a rotation system (T-cube DC Servo controller, Thor labs) to achieve uniform irradiation of the $\mathrm{Ag}$ surface.

\subsection{Chemical analysis}

ICP-OES (ICAP 6300 duo thermo scientific) was used to determine the amount of $\mathrm{Ag}$ and $\mathrm{Au}$ atoms, in the resulting colloidal solution. For this measurement, $100 \mu \mathrm{L}$ solution of NPs colloidal solution was introduced in aqua regia, and after overnight acid digestion the final volume was adjusted with Milli-Q water to $25 \mathrm{~mL}$. The dilution factor is kept into consideration while determining the final concentration.

\subsection{Optical spectroscopy}

Optical absorption spectra were recorded in a quartz cuvette (10 mm, Helma), using a Cary 6000 UV-VIS double beam spectrophotometer. The scan range was $200-800 \mathrm{~nm}$ with a $600 \mathrm{~nm} \min ^{-1}$ rate.

\subsection{Transmission electron microscopy}

The samples were prepared by dropping dilute solutions of NPs onto carbon coated copper grids, placed in a pumping station and the solvent was allowed to evaporate. The size distribution of nanoparticles was investigated by both conventional TEM and high angular annular dark field (HAADF) imaging in the scanning TEM (STEM) mode. TEM measurements were performed with a JEOL Jem1011 microscope working at an acceleration voltage of $100 \mathrm{kV}$. HAADF-STEM measurements were performed with a JEOL JEM-2200FS microscope, equipped with a field emission gun working at an accelerating voltage of $200 \mathrm{kV}$. The chemical composition of several NPs was determined by Energy Dispersive X-ray Spectroscopy (EDS) analysis performed in the STEM mode, with a JED-2300 $\mathrm{Si}(\mathrm{Li})$ detector and using an electron probe of $0.7 \AA$. The chemical quantification was calculated considering $\mathrm{Ag} \mathrm{L} \alpha$ and $\mathrm{Au} \mathrm{M} \alpha \mathrm{X}$-ray lines for silver and gold, respectively, and using the Cliff-Lorimer method, which is considered as a good approximation for thin specimens.

\subsection{Raman spectroscopy}

All the metal NPs, produced by pulsed laser ablation in liquid (PLAL) were diluted $40 \times$ of the initial concentration in order to have very few molecules available for investigation. psLA-NPs $\left(1.25 \times 10^{-10} \mathrm{M}, V_{\mathrm{NPs}}: 2 \mu \mathrm{L}\right)$ are deposited on the $\mathrm{CaF}_{2}$ substrate and then $\mathrm{CV}$ molecules $\left(1 \mathrm{nM}, V_{\mathrm{CV} / \mathrm{Rd} 6 \mathrm{G}}: 5 \mu \mathrm{L}\right)$ were dropped over the dried area of $p s L A-N P s$. After 20 min reaction time for 
chemisorption, the sample was rinsed with water in order to remove all the $\mathrm{CV}$ molecules, which are not attached to metalNPs. First of all, background measurements were performed for all kind of NPs, produced by PLAL ( $p s L A-N P s$ ) and produced by chemical technique (chem-NPs), without any molecules using $633 \mathrm{~nm}$ laser line. Chem-NPs have been prepared using conventional chemical synthesis for obtaining $15 \mathrm{~nm}$ citrate coated Au NPs. ${ }^{33}$ After the molecule adsorption, the system is ready for SERS investigations.

Microprobed Raman spectra were obtained using a Renishaw inVia Raman microscope at room temperature through a $150 \times$ objective of a Leica microscope. Raman spectra were excited by $532(0.30 \mathrm{~mW}, 5 \mathrm{~s}), 633(0.175 \mathrm{~mW}, 5 \mathrm{~s})$ and 830 $(15 \mathrm{~mW}, 10 \mathrm{~s}) \mathrm{nm}$ from a solid state, He-Ne and diode laser, respectively, in backscattering geometry. The experimental parameters are fixed unless otherwise mentioned specifically. Two different gratings, 1200 and 1800 lines per mm, were used for the $830 \mathrm{~nm}$ laser, and 532 and $633 \mathrm{~nm}$ laser, respectively. At most the 3rd order polynomial baseline correction was performed without any further data processing. In this manuscript, a static experimental range (for $532 \mathrm{~nm}$ laser, $280-2014 \mathrm{~cm}^{-1}$; for $633 \mathrm{~nm}$ laser, $385-1551 \mathrm{~cm}^{-1}$ whereas for $830 \mathrm{~nm}$ laser, $359-1380 \mathrm{~cm}^{-1}$ ) is used for each laser excitation wavelength.

\subsection{XRD structural analysis}

XRD spectra were recorded on a Smartlab $9 \mathrm{~kW}$ Rigaku diffractometer, equipped with a copper rotating anode. The X-ray source was operated at $40 \mathrm{kV}$ and $150 \mathrm{~mA}$. A Gobel mirror was used to obtain a parallel beam and to suppress $\mathrm{Cu} K \beta$ radiation (1.392 $\AA$ ). The measurements were performed using a $2 \theta$ scan.

\section{Results and discussion}

\subsection{Morphologies}

Au NPs colloidal solution was prepared by laser ablation on a $\mathrm{Au}$ target in deionized water. The ligand-free Au NPs are nearly spherical in shape with diameters of $8 \pm 5 \mathrm{~nm}$ (Fig. S1, ESI $\dagger$ ). Fig. 1 shows typical TEM images with the corresponding size histogram of colloidal solution obtained by PLAL of the Ag target placed in a glass filled with a $80 \mathrm{ppm} \mathrm{Au} \mathrm{colloidal}$ solution for different wavelength irradiation. For all laser excitation, we can observe that generated NPs have a pseudospherical morphology and smooth surface. The mean diameter is found to be below $20 \mathrm{~nm}$, i.e. 5, 15 and $8.5 \mathrm{~nm}$ using $355 \mathrm{~nm}$, $532 \mathrm{~nm}$ and $1064 \mathrm{~nm}$ laser wavelength excitation, respectively. STEM-EDS analyses of several AuAg NPs, obtained by using all excitation laser wavelengths, were acquired systematically to assess their chemical composition. Fig. 2a shows the HAADF STEM image and STEM-EDS line profile of AuAg NPs synthesized by $355 \mathrm{~nm}$ laser ablation in $80 \mathrm{ppm}$ Au solution. STEMEDS elemental analysis confirmed the formation of $\mathrm{AuAg}$ homogeneous alloys NPs, where the content of the Ag end-member was varying from $15 \%$ to $25 \%$ (at.) in agreement with initial $\mathrm{Au}$ concentration in the solution. The elemental distribution of $\mathrm{Ag}$ and $\mathrm{Au}$ across NPs was not consistent with core-shell structure and no Ag or Au single phases NPs were

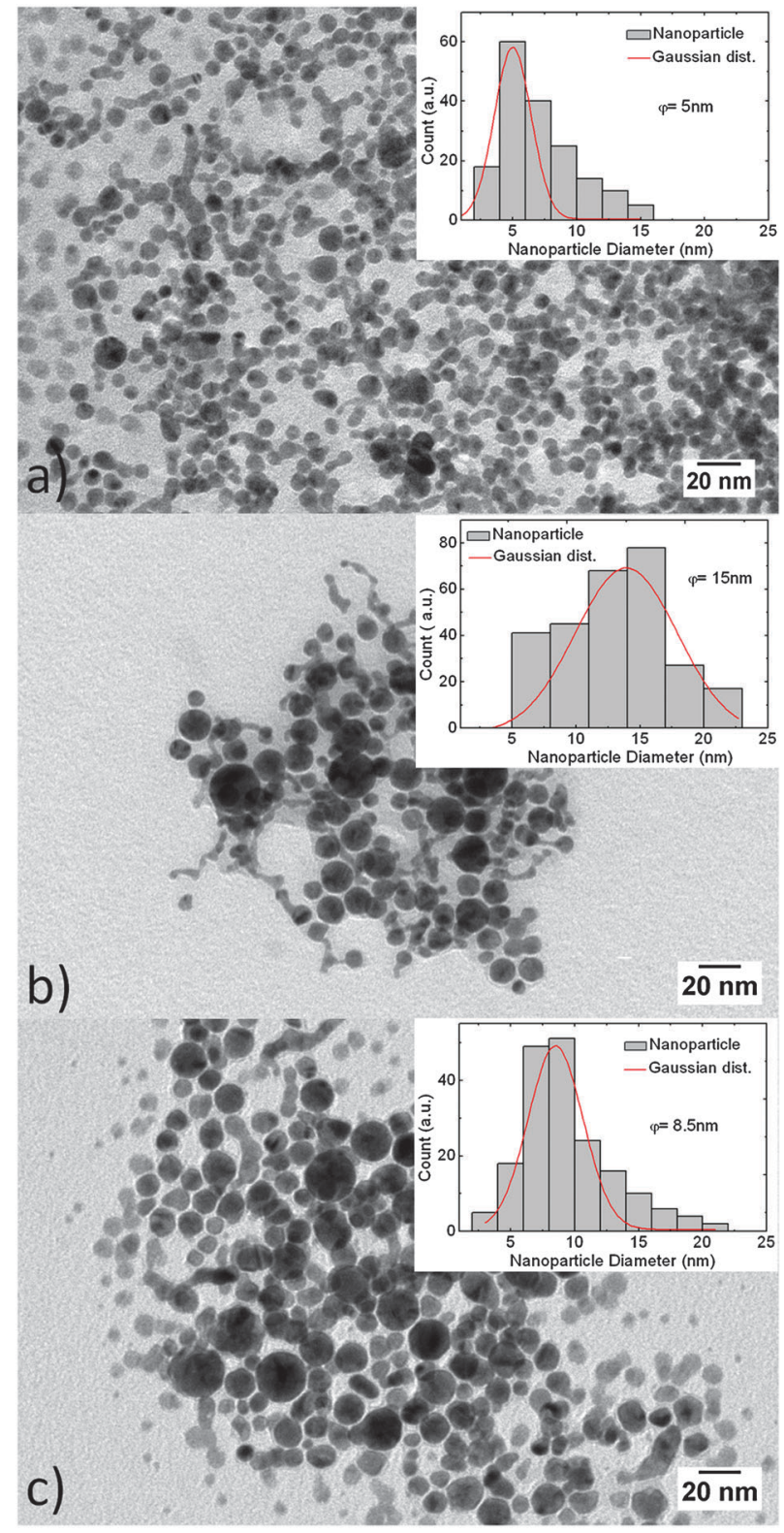

Fig. 1 TEM images of metallic NPs solution obtained by PLAL of Ag target placed in a ligand-free Au NPs solution for different irradiation wavelength: (a) $355 \mathrm{~nm}$, (b) $532 \mathrm{~nm}$ and (c) $1064 \mathrm{~nm}$.

observed. It might also be noted that the $\mathrm{Ag}$ content of as-obtained alloy NPs is controlled by the variation of the Au NPs concentration in the mother solution, as we will described in detail in the next section. On the other hand, STEM-EDS analysis on NPs, produced with $1064 \mathrm{~nm}$ laser excitation, revealed chemical compositions consistent with metallic $\mathrm{Ag}$ and $\mathrm{Au}$ NPs, and not with alloys of these two end-members (not reported data). XRD investigations of bimetallic NPs exhibited their crystalline nature displaying diffraction peaks compatible with both pure $\mathrm{Au}$ and $\mathrm{Ag}$ single phases, owing to the structural similarities between $\mathrm{Au}$ and $\mathrm{Ag}$ phases in terms of lattice parameters $\left(a_{\mathrm{Ag}}=4.086 \AA\right.$; $\left.a_{\mathrm{Au}}=4.078 \AA\right)$ and space groups (cubic $F m \overline{3} m$ ) (Fig. 2b). 

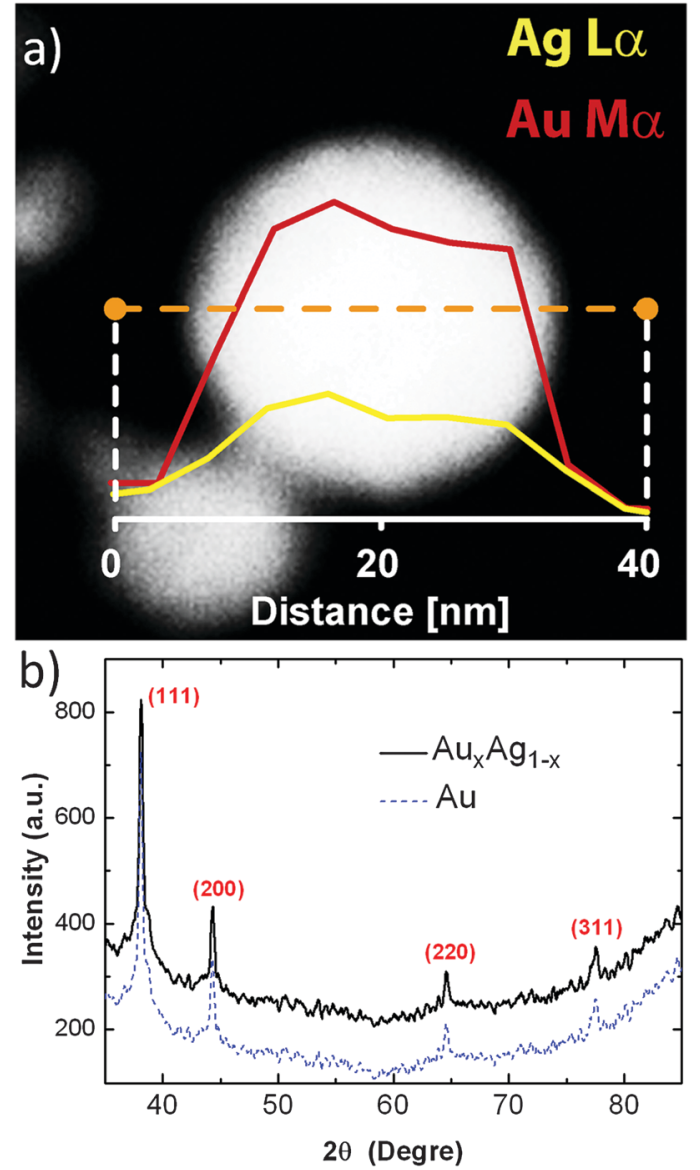

Fig. 2 (a) HAADF STEM image of AuAg bimetallic NPs and the EDS line profile (orange line) showing the elemental distribution of $\mathrm{Au}$ (M $\alpha$-edge) and $\mathrm{Ag}$ (La-edge). (b) XRD pattern of AuAg bimetallic NPs exhibiting the (111), (200), (220) and (311) main peaks of cubic Au and Ag phases. Blue dashed line corresponds to XRD pattern of Au NPs.

\subsection{Optical properties}

Fig. 3 shows the normalized absorption spectra of the resulting colloidal solution, prepared by PLAL in Au NPs solutions of different concentrations. Two distinct behaviors could be noticed with the irradiation wavelength. The plasmon peak position is found to shift covering the visible range located between $400 \mathrm{~nm}$ (Ag plasmon) and $520 \mathrm{~nm}$ (Au plasmon) with the increase of the Au concentration in solution, when the second and third harmonic laser lines were used. Such a behavior is in agreement with previous bimetallic NPs formation by laser irradiation of metallic colloidal solution ${ }^{29,30}$ and the chemical reduction method. ${ }^{18}$ In the Fig. $4 \mathrm{a}$, the plasmon peak position shift rate, estimated by a linear fitting, reveals that the plasmonic resonance tuning effect is more efficient using third harmonic irradiation of the ps laser system with respect to the first and second harmonic. The plasmon peak position shift rate value is $0.72 \pm 0.15 \mathrm{~nm}$ per percent and $1.21 \pm 0.06 \mathrm{~nm}$ per percent for the second and third harmonic excitation wavelength, respectively. On the other hand, the resulting colloidal solution obtained by ablation using the fundamental harmonic wavelength exhibits two distinct



Fig. 3 Normalized absorption spectra of metallic NPs solution prepared in Au NPs solution of different concentrations at different wavelength irradiation (a) $355 \mathrm{~nm}$, (b) $532 \mathrm{~nm}$ and (c) $1064 \mathrm{~nm}$.

absorbance peaks at $400 \mathrm{~nm}$ and $526 \mathrm{~nm}$, corresponding to $\mathrm{Ag}$ and $\mathrm{Au}$ surface plasmon bands respectively. As the gold concentration in solution increases, both plasmon resonance peak positions remain unchanged which indicates that the obtained colloidal solution is a mixture of Au and Ag NPs in agreement with previous chemical analysis. Therefore, in contrast to ablation with the second and third harmonic beams, using the fundamental beam the alloying process does not occur in our experimental conditions.

\subsection{Alloying process}

In a previous report the synthesis of bimetallic NPs and the tuning of plasmonic response i.e. tuning of the alloy composition were controlled by adjusting both initial concentration of $\mathrm{Ag}$ and $\mathrm{Au}$ colloids mixture, and/or the irradiation time. ${ }^{29-31} \mathrm{In}$ our laser-based approach consisting of the ablation of a $\mathrm{Ag}$ target in Au colloidal solution, only one mono metallic reactant amount is defined, Au NP, while Ag elemental concentration is unknown, prior to ablation. Therefore we measured the amount of the $\mathrm{Ag}$ atom in the as prepared colloidal solutions by the ICP-OES technique. Fig. $4 \mathrm{~b}$ shows the $\mathrm{Ag}$ yield produced as a function of Au NPs concentration in solution for different wavelength irradiation. Chemical analysis reveals that the $\mathrm{Ag}$ atom yield decreases with an increase of Au concentration in solution using the second and third harmonic while the 

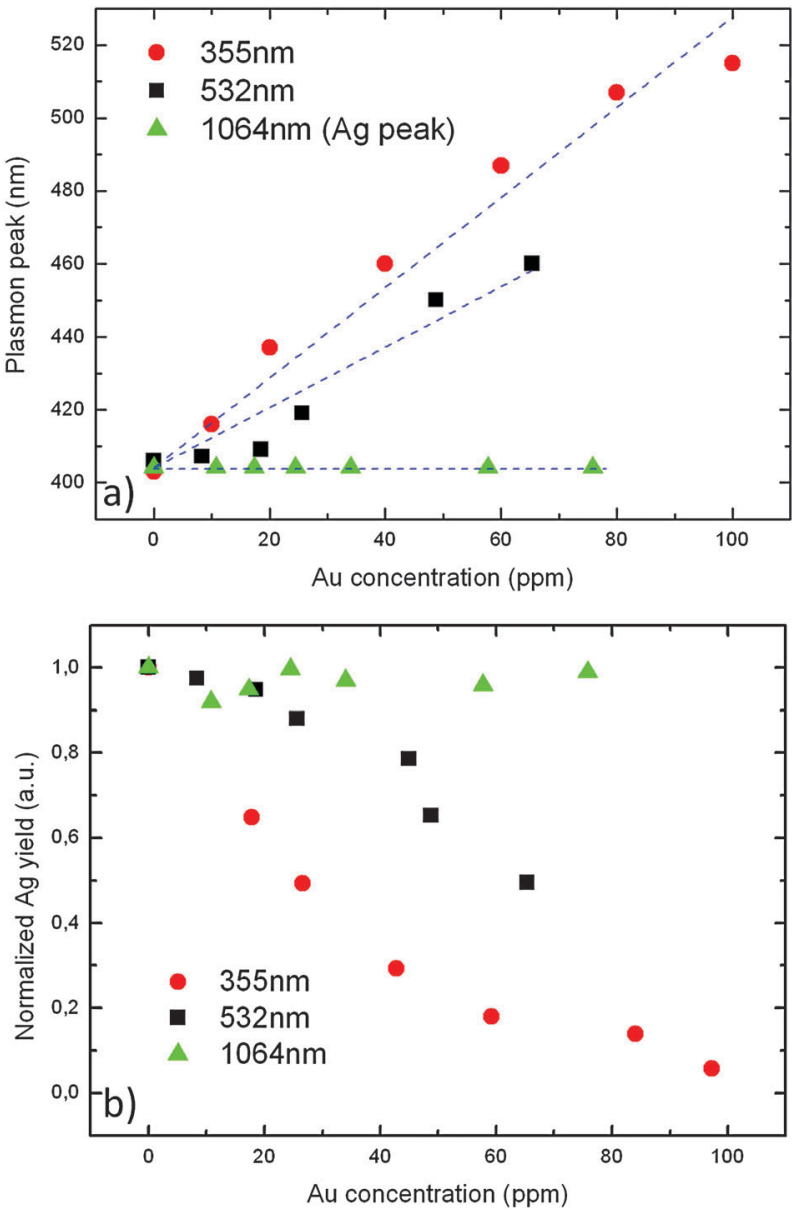

Fig. 4 (a) Variation of the plasmon peak position of the metallic NPs solution as a function of Au NPs concentration in solution obtained at different wavelength irradiation. (b) Normalized silver atom yield generated as a function of $\mathrm{Au}$ concentration in solution for different wavelength irradiation.

amount of Ag atom is constant for infrared wavelength irradiation. For instance, the use of $355 \mathrm{~nm}$ irradiation wavelength induced the generation of $20 \mathrm{ppm} \mathrm{Ag}$ atom in a solution of 80 ppm Au atom, leading to the generation of bimetallic NPs with molar ratio 1/4 Ag/Au with a plasmon resonance peak at $508 \mathrm{~nm}$ (Fig. 3a).

In contrast to other works, we demonstrate here that the Ag atoms yield, i.e. bimetallic NPs composition, depends on the initial $\mathrm{Au}$ concentration in solution and laser wavelength irradiation used for ablation. The generated $\mathrm{Ag}$ atom yield, i.e. the bimetallic NPs composition, is explained by an in situ laser shielding effect which is an interaction of the laser light with colloidal solution. ${ }^{34,35}$ The ablation using the second, $532 \mathrm{~nm}$, and third, $355 \mathrm{~nm}$, harmonic is affected by the losses of excitation light reaching the target surface due to interaction between the surface plasmonic resonance of the Au colloidal solution and laser irradiation, resulting in a decrease of $\mathrm{Ag}$ yield with an increase of Au concentration. In the mean time, physicochemical phenomena like laser-induced reduction, melting and fragmentation, followed by a spontaneous atom inter-diffusion process, occur leading to nanoalloy formation. ${ }^{29-32}$
While, using the fundamental laser wavelength at $1064 \mathrm{~nm}$, there is no interaction between the laser beam and the Au NPs colloidal solution. Therefore, loss of excitation light is weak, ${ }^{34}$ and the laser power on the target is constant, resulting in a constant Ag yield in agreement with optical analysis (Fig. 3c and 4a). The absence of interaction between the fundamental laser beam and the AU NPs results in less efficient fragmentation and melting, and therefore the alloying process cannot occur.

\subsection{SERS applications}

Various SERS research works were reported on different metal NPs, produced by chemical techniques. The impurity present during the chemical reaction to form NPs may hinder the adsorption of molecules on the surface or for labeling the NPs because of the residual ligands on the surface of metal NPs. The background Raman measurements over all the metal NPs, produced by psLA-NPs and produced by chem-NPs (diluted $40 \times$ of the initial concentration), were investigated using a $633 \mathrm{~nm}$ laser line. SERS background measurements for metallic NPs produced by two different routes are shown in Fig. 5 in the range of $500-3200 \mathrm{~cm}^{-1}$. Raman spectrum of $p s L A-N P s$ shows a featureless spectrum without any Raman band throughout the experimental range. Nevertheless, Raman spectrum of chem-NPs displays; (a) a high fluorescence background, and (b) various Raman bands, in particular, a broad band in the range of 2800-3100 $\mathrm{cm}^{-1}$, associated to the different $\mathrm{C}-\mathrm{H}_{x}$ stretching vibrational band. ${ }^{36,37}$ These vibrational bands occur due to the presence of contaminants. It should be noticed that though the laser power is kept constant $(0.27 \mathrm{~mW})$ for both kind of NPs, the accumulation time for chem-NPs is $10 \mathrm{~s}$, whereas, for psLA-NPs, it is $30 \mathrm{~s}$. From Fig. 5, it is clear that though the accumulation time is longer for $p s L A-N P s$, the spectrum background is much lower and featureless, demonstrating perfect SERS NPs. The major advantages of psLA-NPs over chem-NPs are that these NPs are ligand-free, stable and reproducible. Being ligand-free NPs, $p s L A-N P S$ provide maximum possibility for the adsorption of molecules/labeling the NPs.

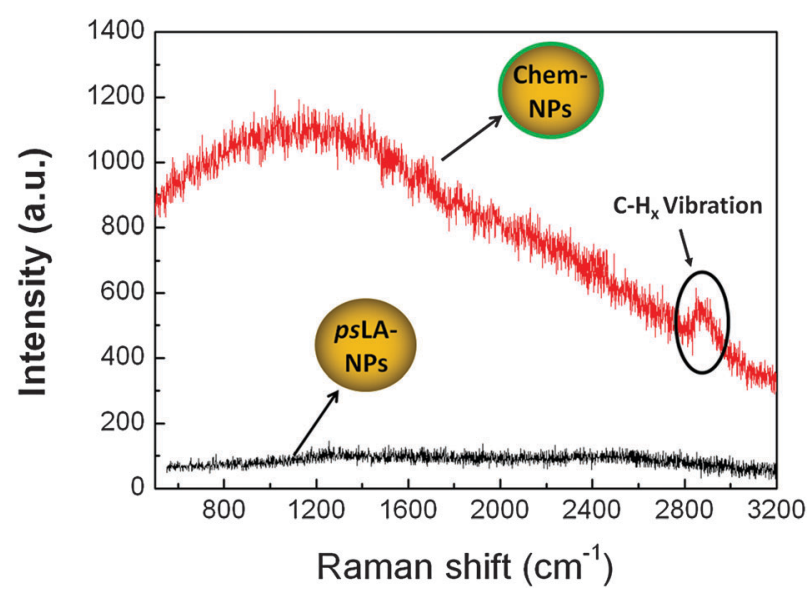

Fig. 5 Raman spectra of metallic NPs, produced by PLAL (psLA-NPs, ligand-free surface), and produced by chemical method (chem-NPs, citrate capped surface). 

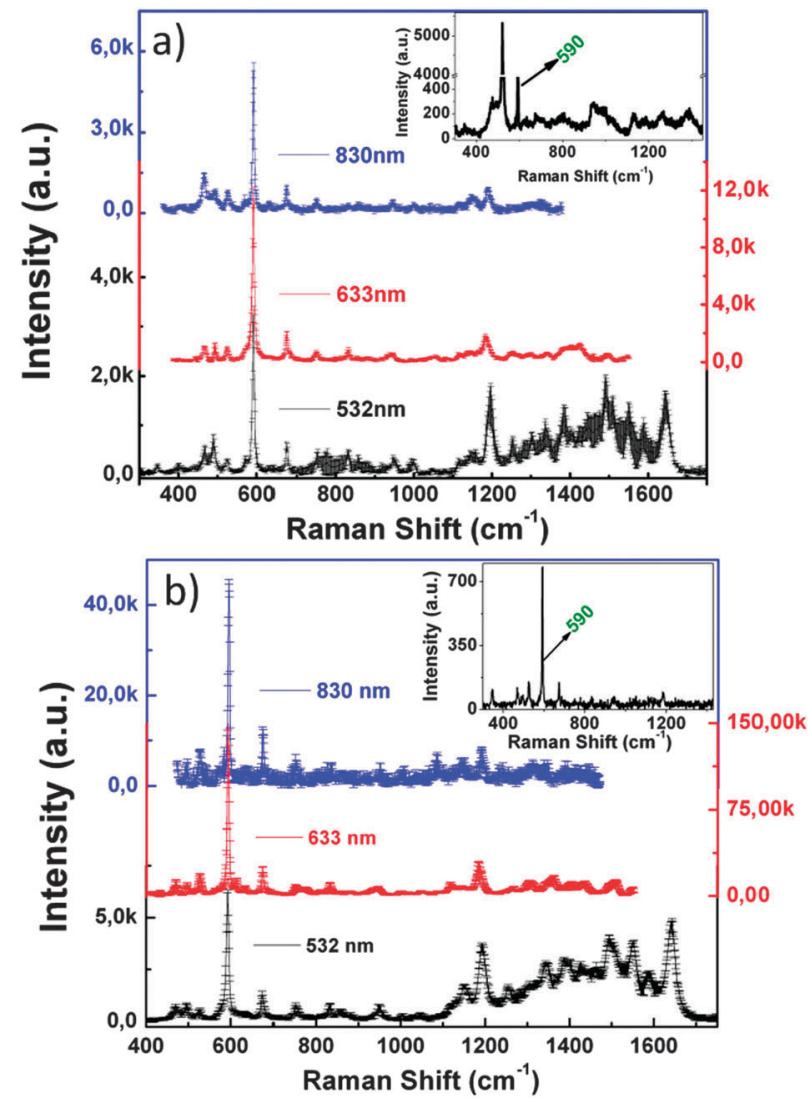

Fig. 6 SERS spectra of CV (1 nM) on LA-NPs, deposited over (a) AuAg NPs and (b) $\mathrm{Au}$ NPs. The measurements were also performed by varying the laser wavelengths $(532,633$ and $830 \mathrm{~nm})$. In the inset, the control measurements, CV deposited over the flat metal surface, is also shown. For the flat AuAg surface, $P_{\text {laser }}=350 \mu \mathrm{W} ; t=30 \mathrm{~s}$, whereas for the flat Au surface, $P_{\text {laser }}=35 \mu \mathrm{W} ; t=50 \mathrm{~s}$.

$\mathrm{CV}$ is an organic dye, intensively employed in biology and medicine, as a histological stain. It is an effective stain used for highlighting acidic components of tissues and is frequently used for nerve tissue areas. SERS spectra in the range of $400-1750 \mathrm{~cm}^{-1}$ of $\mathrm{CV}$, deposited over AuAg NPs and over $\mathrm{Au}$ NPs, are shown in Fig. 6 for different laser excitation wavelength. Various characteristic Raman bands of CV molecules are clearly observed ${ }^{38}$ for all the laser excitation wavelength lines. A strong and intense characteristic peak, centred at 591 $\mathrm{cm}^{-1}$, is clearly observed. Many interesting aspects are revealed from these measurements (Fig. 6) such as the variation in different band intensity, the ratio of vibrational bands, etc. In Fig. 6a, SERS spectra of CV, excited by varying laser wavelengths, deposited over AuAg psLA-NPs are shown. In the inset, the control measurement of $\mathrm{CV}$, deposited on a flat AuAg surface, is also reported. One intense band at $591 \mathrm{~cm}^{-1}$ and low intense characteristic bands, centered at around 675, 1186 and $1640 \mathrm{~cm}^{-1}$, were observed which could be associated to the combination of in-plane $\mathrm{N}-\mathrm{H}_{2}$ and ring bending, ring deformation, combination of $\mathrm{N}-\mathrm{H}_{2}$ rocking and $\mathrm{C}-\mathrm{H}_{x}$ rocking, and combination of ring stretching, $\mathrm{C}-\mathrm{O}-\mathrm{C}$ bending and $\mathrm{C}-\mathrm{N}-\mathrm{C}$ symmetric stretching, respectively. ${ }^{38,39}$ In the same inset (Fig. 6a), one strong band, centered at around $520 \mathrm{~cm}^{-1}$, is
Table 1 The SERS band intensity for 591 and $1190 \mathrm{~cm}^{-1}$ and their ratio are reported for AuAg and Au psLA-NPS

\begin{tabular}{lrrl}
\hline & Intensity & \\
\cline { 2 - 4 }$\lambda(\mathrm{nm})$ & \multicolumn{1}{c}{$I_{591}$} & $I_{1189}$ & $I_{591} / I_{1189}$ \\
\hline $\mathrm{AuAg} p s L A-N P S$ & & 910 & \\
830 & 5294 & 1730 & 5.81 \\
633 & 11490 & 1400 & 6.64 \\
532 & 3245 & & 2.31 \\
$\mathrm{Au} p$ pLA-NPS & & 8473 & \\
830 & 45155 & 25629 & 5.32 \\
633 & 143074 & 3572 & 5.58 \\
532 & 5723 & & 1.60 \\
\hline
\end{tabular}

also observed which is the contribution from the Si substrate beneath the flat AuAg metal surface. It can be observed that the Raman bands of CV, in the range of $1100-1700 \mathrm{~cm}^{-1}$, enhance when it was excited by $532 \mathrm{~nm}$ with respect to the other laser lines i.e. 633 and $830 \mathrm{~nm}$. The measurements were also performed for the Au psLA-NPs by varying the laser excitation wavelengths (Fig. 6b). The reference SERS spectra of CV, deposited over the flat Au surface, are also shown in the inset of Fig. 6b. Spectra show the similar trend for CV molecules. Since, the optical absorption band of $\mathrm{CV}$ and the plasmonic resonance peak for Au-NPs are at around 585 and $520 \mathrm{~nm}$, respectively, ${ }^{40}$ the excitation wavelength $(532 \mathrm{~nm})$ is on the resonance of the plasmonic band of Au-NPs and also fulfilling the resonance Raman condition $\left(\sigma_{\text {Raman }} \alpha\left(\lambda_{\text {laser }}\right)^{-4}\right)$. However, the SERS intensity is found to be in its best condition for $633 \mathrm{~nm}$ as far as the vibrational Raman band at $591 \mathrm{~cm}^{-1}$ is concerned. This could be due to the molecular resonance excitation. Hence, in this case, the chemical effect of SERS phenomena plays a remarkable role. In the past, few research works were carried out for the nanoalloy NPs with varying AuAg content, produced by the chemical technique, but nothing has been found, ${ }^{41,42}$ to our knowledge, regarding any SERS study on nanoalloy NPs, produced by laser ablation. Furthermore, the intensity of two characteristic bands centered at 591 and $1189 \mathrm{~cm}^{-1}$ and the band ratio are reported in Table 1. The ratio of two bands decreases when the molecules are being excited with $532 \mathrm{~nm}$. This could be explained by the fact that the average enhancement factor in the SERS phenomena has two foremost contributions from the plasmon resonance: (a) the laser wavelength and (b) the absolute Stokes wavelength. ${ }^{43}$ Indeed, for laser excitation wavelength at $532 \mathrm{~nm}$, the Stokes frequency for Raman shift in the range of $1000-1700 \mathrm{~cm}^{-1}$ is associated with an absolute value around $560-585 \mathrm{~nm}$. Since, the molecular absorption is maximum in this range, the molecules, in fact, sense in the resonant conditions and, consequently, the phenomenon is no more normal SERS but résonance SERS. ${ }^{43-45}$

From SERS spectra of CV, deposited over different psLA-NPS, shown in Fig. 6(a) and (b), the enhancement factor $G$,

$$
G=\frac{\left(I_{\text {SERS }} / N_{\text {SERS }}\right)}{\left(I_{\text {Raman }} / N_{\text {Raman }}\right)}
$$

can be calculated, where $I$ and $N$ are related to the intensity and number of molecules, respectively. The subscripts, SERS and 




Fig. 7 SERS spectrum of Rd6G, deposited over AuAg psLA-NPs, is shown. In the inset, Rd6G deposited over the flat metal surface is also shown. The experimental parameters under both the conditions are also reported.

Raman, are associated to the SERS and Raman measurements, respectively. As explained above the SERS enhancement factor varies from band to band. But, considering that both the NPs show the max enhancement for $633 \mathrm{~nm}$, the calculation was done for the band centred at $591 \mathrm{~cm}^{-1}$. SERS enhancement factors are found to be $7.0 \times 10^{5}$ and $5.7 \times 10^{5}$ for Au NPs and for AuAg NPs with respect to the flat gold and bimetallic surfaces, respectively. The enhancement factor calculation was done with the estimation of a mean diameter of $10 \mathrm{~nm}$. The other values of the experimental parameters can be found in the experimental part and also from Fig. 6. Though the excitation wavelength was not the best one for AuAg NPs, the enhancement factor is found to be very close to Au NPs.

Moreover, SERS experiments were also carried out for bimetallic NPs samples on which Rd6G molecules were chemisorbed. Rd6G molecules, widely used in biotechnology, show the absorption band centered at around $527 \mathrm{~nm}$, which will be relatively out of molecular resonance for $\mathrm{Au} / \mathrm{AuAg}$ NPs with respect to the $\mathrm{CV}$ molecule. The molecular deposition procedure was followed as that of $\mathrm{CV}$, described in the experimental section. The SERS spectrum of the Rd6G molecule, in the experimental range of $750-1750 \mathrm{~cm}^{-1}$, is shown in Fig. 7. Various characteristic bands for Rd6G, centred at 775, 1181, 1310, 1360, 1508 and $1650 \mathrm{~cm}^{-1}$, can be clearly observed which can be attributed to the out of plane $\mathrm{C}-\mathrm{H}$ bending, combination of xanthene ring deformation, $\mathrm{C}-\mathrm{H}$ bending and $\mathrm{N}-\mathrm{H}$ bending, combination of in-plane xanthene ring bending, $\mathrm{C}-\mathrm{H}_{2}$ wagging and $\mathrm{N}-\mathrm{H}$ bending, xanthene ring stretching, stretching vibration of the phenyl ring, and asymmetric stretching of the xanthene ring. ${ }^{4-48}$ In the inset, the reference Rd6G spectrum is also shown. As can be observed from the experimental parameters reported in Fig. 7 for both control and sample measurements, the NPs show a remarkable SERS enhancement. Indeed, when the laser power is 10 times lower and the accumulation time almost half, the SERS intensity of the Rd6G molecule for AuAg NPs reaches about 3500 whereas in the case of the reference measurement it is almost not evident.

\section{Conclusion}

We reported synthesis of ligand-free AuAg bimetallic NPs, by picosecond pulsed laser ablation of $\mathrm{Ag}$ target in $\mathrm{Au}$ NPs suspensions. The relative amount of $\mathrm{Ag}$ is controlled in situ through a laser shielding effect. In opposite to other works, we demonstrated here that the bimetallic NPs composition depends on the initial Au concentration in solution and laser wavelength irradiation used for ablation. Under our experimental conditions, ligand-free AuAg bimetallic NPs are successfully synthesized with tunable localized surface plasmon resonance frequency through with $355 \mathrm{~nm}$ and $532 \mathrm{~nm}$ picosecond laser irradiation in contrast to the $1064 \mathrm{~nm}$ picoseconds laser irradiation. The laser shielding based PLAL approach thus allows the generation of bimetallic NPs of various compositions which could be extended for the production of other bimetallic NPs materials. The unique surface chemistry of bimetallic NPs synthesized by the PLAL method with respect to the chemically synthesized one has been investigated. Ligand-free bimetallic NPs display a featureless Raman background spectrum. The enhancement factor for AuAg NPs is estimated to be about $5.7 \times 10^{5}$ with respect to the flat AuAg surface. Since, the nanoalloy composition is very much critical to have the maximum SERS enhancement, a systematic SERS research with varying AuAg compositions is required.

\section{Acknowledgements}

This work was supported by the Italiano Istituto di Tecnologia. The authors gratefully acknowledge the technical help of Marco Scotto d' Abbusco and Dr Andreas Riedinger for chemical synthesis of metallic nanoparticles.

\section{References}

1 J. N. Anker, W. P. Hall, O. Lyandres, N. C. Shah, J. Zhao and R. P. Van Duyne, Nat. Mater., 2008, 7, 442-453.

2 A. J. Haes, L. Chang, W. L. Klein and R. P. Van Duyne, J. Am. Chem. Soc., 2005, 127, 2264-2271.

3 J. Yguerabide and E. E. Yguerabide, Anal. Biochem., 1998, 262, 137-156.

4 J. M. Nam, C. S. Thaxton and C. A. Mirkin, Science, 2003, 301, 1884-1886.

5 C. Eliasson, A. LorÉn, J. Engelbrektsson, M. Josefson, J. Abrahamsson and K. Abrahamsson, Spectrochim. Acta, Part A, $2005,61,755-760$.

6 H. A. Atwater and A. Polman, Nat. Mater., 2010, 9, 205-213.

7 A. Q. Wang, C. M. Chang and C. Y. Mou, J. Phys. Chem. B, 2005, 109, 18860-18867.

8 A. Q. Wang, J. H. Liu, S. D. Lin, T. S. Lin and C. Y. Mou, J. Catal., 2005, 233, 186-197.

9 P. Mulvaney, Langmuir, 1996, 12, 788-800.

10 S. M. Stranahan and K. A. Willets, Nano Lett., 2010, 10, 3777-3784. 11 J. N. Anker, W. P. Hall, O. Lyandres, N. C. Shah, J. Zhao and R. P. Van Duyne, Nat. Mater., 2008, 7, 442-453.

12 M. M. Miranda, C. Gellini and E. Giorgetti, J. Phys. Chem. C, 2011, 115, 5021-5027.

13 S. Nie and S. R. Emory, Science, 1997, 275, 1102-1106.

14 K. Kneipp, Y. Wang, H. Kneipp, L. T. Perelman, I. Itzkan, R. R. Dasari and M. S. Feld, Phys. Rev. Lett., 1997, 78, 1667-1670.

15 F. De Angelis, F. Gentile, F. Mecarini, G. Das, M. Moretti, P. Candeloro, M. L. Coluccio, G. Cojoc, A. Accardo, C. Liberale, R. P. Zaccaria, G. Perozziello, L. Tirinato, A. Toma, G. Cuda, R. Cingolani and E. Di Fabrizio, Nat. Photonics, 2011, 5, 682-687.

16 D.-K. Lim, K.-S. Jeon, H. M. Kim, J.-M. Nam and Y. D. Suh, Nat. Mater., 2010, 9, 60-67. 
17 Q. Zhang, C. H. Moran, X. Xia, M. Rycenga, N. Li and Y. Xia, Langmuir, 2012, 28, 9047-9054.

18 S. Link, Z. L. Wang and M. A. El-Sayed, J. Phys. Chem. B, 1999, 103, 3529-3533.

19 M. P. Mallin and C. J. Murphy, Nano Lett., 2002, 2, 1235-1237.

20 M. D. Massich, D. A. Giljohann, A. L. Schmucker, P. C. Patel and C. A. Mirkin, ACS Nano, 2010, 4, 5641-5646.

21 G. Maiorano, S. Sabella, B. Sorce, V. Brunetti, M. A. Malvindi, R. Cingolani and P. P. Pompa, ACS Nano, 2010, 4, 7481-7491.

22 S. Sabella, A. Galeone, G. Vecchio, R. Cingolani and P. P. Pompa, J. Nanosci. Lett., 2011, 1, 145-165.

23 V. Amendola and M. Meneghetti, Phys. Chem. Chem. Phys., 2009, 11, 3805-3821.

24 R. Intartaglia, K. Bagga, M. Scotto, A. Diaspro and F. Brandi, Opt. Mater. Express, 2012, 2, 510-518.

25 R. Intartaglia, K. Bagga, F. Brandi, A. Genovese, E. Di Fabrizio and A. Diaspro, J. Phys. Chem. C, 2011, 115, 5102-5107.

26 R. Intartaglia, K. Bagga, A. Genovese, A. Athanassiou, R. Cingolani, A. Diaspro and F. Brandi, Phys. Chem. Chem. Phys., 2012, 14, 15406-15411.

27 R. Intartaglia, A. Barchanski, K. Bagga, A. Genovese, G. Das, P. Wagener, E. Di Fabrizio, A. Diaspro, F. Brandi and S. Barcikowski, Nanoscale, 2012, 4, 1271-1274.

28 J. G. Walter, S. Petersen, F. Stahl, T. Scheper and S. Barcikowski, J. Nanobiotechnol., 2010, 8, 21-27.

29 S. Besner and M. Meunier, J. Phys. Chem. C, 2011, 114, 10403-10409.

30 G. Compagnini, E. Messina, O. Puglisi, R. S. Cataliotti and V. Nicolosi, Chem. Phys. Lett., 2008, 457, 386-390.

31 P. Jafarkhani, M. J. Torkamany, S. Dadras, A. Chehrghani and J. Sabbaghzadeh, Nanotechnology, 2011, 22, 235703-235710.

32 R. Kuladeep, L. Jyothi, K. Shadak Alee, K. L. N. Deepak and D. Narayana Rao, Opt. Mater. Express, 2012, 2, 161-172.
33 J. Turkevich, P. C. Stevenson and J. A. Hillier, Discuss. Faraday Soc., 1951, 11, 55-75.

34 A. Schwenke, P. Wagener, S. Nolte and S. Barcikowski, Appl. Phys. A: Mater. Sci. Process., 2011, 104, 77-82.

35 F. Mafune, J. Y. Kohno, Y. Takeda, H. Sawabe and T. Kondow, J. Phys. Chem. B, 2001, 105, 5114-5120.

36 G. Das, G. Mariotto and A. Quaranta, J. Electrochem. Soc., 2006, 153, F46-F51.

37 A. Majumdar, G. Das, N. Patel, P. Mishra, D. Ghose and R. Hippler, J. Electrochem. Soc., 2008, 155, D22-D26.

38 E. Vogel, A. Gbureck and W. Kiefer, J. Mol. Struct., 2000, 550, 177-190.

39 A. Kudelski, Chem. Phys. Lett., 2005, 414, 271-275.

40 G. Das, N. Patra, A. Gopalakrishana, R. P. Zaccaria, A. Toma, S. Thorat, E. Di Fabrizio, A. Diaspro and M. Salerno, Analyst, 2012, 137, 1785-1792.

41 Y. Yang, J. Shi, G. Kawamura and M. Nogami, Scr. Mater., 2008, 58, 862-865.

42 Y. Cui, B. Ren, J. Yao, R. A. Gu and Z. Q. Tian, J. Phys. Chem. B, 2006, 110, 4002-4006.

43 E. C. Le Ru, E. Blackie, M. Meyer and P. G. Etchegoin, J. Phys. Chem. C, 2007, 111, 13794-13803.

44 A. Bonifacio, L. van der Sneppen, C. Gooijer and G. van der Zwan, Langmuir, 2004, 20, 5855-5864.

45 F. T. Docherty, P. B. Monaghan, R. Keir, D. Graham, W. E. Smith and J. M. Cooper, Chem. Commun., 2004, 118-119.

46 M. L. Coluccioa, G. Das, F. Mecarini, F. Gentile, A. Pujia, L. Bava, R. Tallerico, P. Candeloro, C. Liberale, F. De Angelis and E. Di Fabrizio, Microelectron. Eng., 2009, 86, 1085-1088.

47 J. Chowdhury, P. Pal, M. Ghosh and T. N. Misra, J. Colloid Interface Sci., 2001, 235, 317-324.

48 L. Jensen and G. C. Schatz, J. Phys. Chem. A, 2006, 110, 5973-5977. 